$\xi=1$ 圆

\title{
Time Control Chart for Two Inverted Models
}

\author{
R. Subba Rao ${ }^{*}$, M. Pushpa Latha ${ }^{2}$, R.R.L. Kantam ${ }^{3}$ \\ ${ }^{I}$ Department of Mathematics, S.R.K.R Engineering College, Bhimavaram, Andhra Pradesh, India-534 204 \\ ${ }^{2}$ Department of Mathematics, Vishnu Institute of Technology, Bhimavaram, Andhra Pradesh, India-534 204 \\ ${ }^{3}$ Retd. Prof. of Statistics, Acharya Nagarjuna University, Guntur, Andhra Pradesh, India-522 508 \\ *Corresponding Author Email: rsubbarao9@gmail.com
}

\begin{abstract}
Control charts are one of the powerful techniques of Statistical Process Control. Control charts are widely accepted and applied in industry which can be used to improve productivity, prevent defects and unnecessary process adjustment. Moreover, they also provide information in diagnosis and process capability. Life time data generally contain the failure times of sample products or inter failure times or number of failures experienced in a given time. The time to failure of a product is to be considered as a quality characteristic to assess the quality of the product. Control limits are evaluated for the time to failure. In this paper the time to failure of a product is considered to follow Inverse Rayleigh and Inverse Half Logistic distributions. Life time data are compared with the control limits to judge the quality performance of the product.
\end{abstract}

Keywords: Life time data; M LE; Regression; Time between failures; Time Control Chart.

\section{Introduction}

Life time data generally contain the failure times of sample products or inter failure times or number of failures experienced in a given time. The time to failure of a product is to be considered as a quality characteristic to assess the quality of the product. Control limits are evaluated for the time to failure. Life time data falling below lower control limit, above upper control limit or between the control limits indicate whether the life pattern of the product is performing adversely, encouragingly or satisfactorily. Generally, assuming a suitable probability model for the life time random variable, the reliability of the product is computed using life time data and the quality with respect to reliability would be assessed. From a different point of view, if the specific life time data contain, times between successive failures, also called inter failure times, probability limits for such a data can be constructed in a parametric approach. Points above the upper control limit of such a data would be an encouraging characteristic of the product because they lead to a large gap between successive failures so that the uptime of the product is large. Hence the product is preferable. That is, detection of out of control above the Upper Control Limit (UCL) is desirable and its causes are to be preserved or encouraged. Similarly, detection of out of control below the Lower Control Limit (LCL) results in shorter gaps between successive failures. The assignable causes for this detection are to be minimized or eliminated. Points within the control limits indicate a smooth and satisfactory failure phenomenon. Thus, such a set of control limits would be helpful in assessing the quality of the product based on inter failure time data. The control chart may be accordingly named as Time Control Chart.

Xie et al. (2002) [1] have suggested time control charts for failure data modeled by the well known Exponential distribution. Tadikamalla and Popescu (2007) [2] developed the kurtosis correction method for $\bar{X}$ and $\mathrm{R}$ control charts for Long-Tailed sym- metrical distributions, Subba Rao and Kantam (2008) [3] constructed the variable control charts for process mean with reference to Double Exponential distribution, Ravi Kumar and Kantam (2010) [4] adopted the principle of Xie et al. (2002)[1] to develop time control chart for Gamma distribution and Half Logistic distribution, Chaitanya Priya (2011)[5] developed the kurtosis correction method for variable control charts, Srinivasa Rao et al. (2012) [6] studied the extreme value charts and Analysis of Means based on the Log-Logistic distribution, Kantam and Ravi Kumar (2013) [7] explained the time control chart for Burr distribution, Subba Rao et al. (2016) [8] constructed the skewness corrected control charts for two inverted models.

In the present paper we adopt the same principle to develop time control charts for life time data modeled by the two inverse distributions namely Inverse Rayleigh Distribution (IRD) and Inverse Half Logistic Distribution (IHLD). The rest of the paper is organized as follows. Section-2 deals with the general theory of monitoring time between failures and its application to the Inverse Rayleigh distribution and Inverse Half Logistic distribution. An extension of time control chart for time to every $\mathrm{r}^{\text {th }}$ failure called $\mathrm{t}_{\mathrm{r}} \mathrm{con}$ trol chart is also developed using the cumulative distribution function of highest order statistic in the given subgroup is discussed in Section-3. Comparison and conclusions are presented in Section-4 of this paper.

\section{Monitoring of Time between Failures with Respect to IRD \& IHLD}

Let $\mathrm{F}(\mathrm{x})$ be the cumulative distribution function of a continuous positive valued random variable, $\mathrm{f}(\mathrm{x})$ be its probability density function. If the random variable representing inter failure times of a device (time lapse between successive failures), a control chart for such a data would be based on 0.9973 probability limits (on 
par with the probability content chosen by Shewart for the classical control charts) of the times between failure random variable, say X. These limits and the central line are respectively the solutions of the following equations taking equitailed probabilities.

$\mathrm{F}(\mathrm{x})=0.99865$

$\mathrm{F}(\mathrm{x})=0.5$

$\mathrm{F}(\mathrm{x})=0.00135$

Let $X_{U}, X_{C}, X_{L}$ be respectively the solutions of equations (1), (2) and (3) in the standard form

i.e., $\quad X_{U}=F^{-1}(0.99865)$

$X_{C}=F^{-1}(0.5)$

$X_{L}=F^{-1}(0.00135)$

The graph between the serial number of the failure and corresponding inter failure time together with 3 parallel lines to the horizontal axis at $\mathrm{X}_{\mathrm{U}}, \mathrm{X}_{\mathrm{C}}, \mathrm{X}_{\mathrm{L}}$ is the time control chart. For a ready reference the pdf and cdf of IRD and IHLD are given in equations (7) through (10)

$$
\begin{aligned}
& f(x)=\frac{2}{\lambda^{2} x^{3}} e^{\frac{-1}{\lambda^{2} x^{2}}}, x>0, \lambda>0 \\
& F(x)=e^{\frac{-1}{\lambda^{2} x^{2}}}, x>0, \lambda>0 \\
& f(x)=\frac{2 b e^{-b / x}}{x^{2}\left(1+e^{-b / x}\right)^{2}}, x>0, b>0 \\
& F(x)=\frac{2 e^{-b / x}}{1+e^{-b / x}}, x>0, b>0
\end{aligned}
$$

It can be seen that for IRD the MLE of $\lambda$ is obtained from the formula

$$
\lambda^{\wedge^{2}}=\frac{1}{n} \sum_{i=1}^{n} \frac{1}{x_{i}^{2}}
$$

Using the data values of Aarset (1987) [9] we can get the MLE of $\lambda$ for IRD which in turn will be useful with the help of equations (4), (5) and (6) to get the UCL, CL and LCL of the control chart. However, the parameter ' $b$ ' of IHLD is estimated by a regression approach as described below.

The cumulative distribution function of IHLD when equated to a fraction ' $p$ ' can be rewritten as

$$
x_{i}=\frac{-b}{\ln \left(\frac{p_{i}}{2-p_{i}}\right)}
$$

This can be viewed as $U=b V$

where

$$
\begin{aligned}
& U=x_{i} \\
& V=\frac{-1}{\ln \left(\frac{p_{i}}{2-p_{i}}\right)}
\end{aligned}
$$

which is in the form of a straight line passing through origin. By the regression approach we know that the least squares estimate of ' $b$ ' is given by

$$
\hat{b}=\frac{\sum U V-n \bar{U} \bar{V}}{\sum V^{2}-n(\bar{V})^{2}}
$$

To get $\hat{b}$ we take $x_{i}$ as the $\mathrm{i}^{\text {th }}$ ordered unit of the sample, $p_{i}$ is taken as $\frac{i}{n+1}$. With the estimate of ' $\mathrm{b}$ ' in the cdf of IHLD would give the UCL, CL and LCL for the time control chart when the cumulative distribution function is equated to $0.99865,0.5$ and 0.00135 respectively. The LCL, CL and UCL of IRD and IHLD are presented below with an illustration.

\subsection{Illustration}

Aarset (1987) [9] gives the data representing the life times of 50 devices are given in Table 1 .

Table 1: Life times of 50 devices

\begin{tabular}{|c|c|c|c|c|c|c|c|c|c|}
\hline $\begin{array}{c}\text { S. } \\
\mathbf{N}\end{array}$ & $\begin{array}{c}\text { De- } \\
\text { vices }\end{array}$ & $\begin{array}{c}\text { S. } \\
\mathbf{N} \\
\text { o. }\end{array}$ & $\begin{array}{c}\text { De- } \\
\text { vices }\end{array}$ & $\begin{array}{c}\text { S. } \\
\mathbf{N} \\
\mathbf{o .}\end{array}$ & $\begin{array}{c}\text { De- } \\
\text { vices }\end{array}$ & $\begin{array}{c}\text { S. } \\
\mathbf{N} \\
\mathbf{o .}\end{array}$ & $\begin{array}{c}\text { De- } \\
\text { vices }\end{array}$ & $\begin{array}{c}\text { S. } \\
\mathbf{N} \\
\mathbf{o .}\end{array}$ & $\begin{array}{c}\text { De- } \\
\text { vices }\end{array}$ \\
\hline 1 & 0.1 & 11 & 7 & 21 & 36 & 31 & 67 & 41 & 84 \\
\hline 2 & 0.2 & 12 & 11 & 22 & 40 & 32 & 67 & 42 & 84 \\
\hline 3 & 1 & 13 & 12 & 23 & 45 & 33 & 67 & 43 & 84 \\
\hline 4 & 1 & 14 & 18 & 24 & 46 & 34 & 67 & 44 & 85 \\
\hline 5 & 1 & 15 & 18 & 25 & 47 & 35 & 72 & 45 & 85 \\
\hline 6 & 1 & 16 & 18 & 26 & 50 & 36 & 75 & 46 & 85 \\
\hline 7 & 1 & 17 & 18 & 27 & 55 & 37 & 79 & 47 & 85 \\
\hline 8 & 2 & 18 & 18 & 28 & 60 & 38 & 82 & 48 & 85 \\
\hline 9 & 3 & 19 & 21 & 29 & 63 & 39 & 82 & 49 & 86 \\
\hline 10 & 6 & 20 & 32 & 30 & 63 & 40 & 83 & 50 & 86 \\
\hline
\end{tabular}

Through the QQ plot correlation technique, it is observed that the probability models IRD and IHLD are the good fit for the given example. The control chart constants constructed for the above data by taking IRD and IHLD as probability models are presented in the following Table 2 .

Table 2: Control Limits for Time between Failures

\begin{tabular}{|c|c|c|c|}
\hline Probability Model & LCL & CL & UCL \\
\hline IRD & 0.24085 & 0.74362 & 16.84979 \\
\hline IHLD & 0.54197 & 3.59797 & 1464.00371 \\
\hline
\end{tabular}

\section{3. $t_{r}$ Control Chart}

The control limits and the central line are based on the parameters of the population and can be estimated only from a given data supposed to have been following the population. Sometimes the lapse of time up to $\mathrm{r}^{\text {th }}$ failure becomes a deciding factor about the failure trend in a given sample of inter failure times. If we are given a series of $\mathrm{n}$-inter failure times; let $\mathrm{r}$ be a natural number less than $\mathrm{n}$. The summations $\sum_{i=1}^{r} X_{i}, \sum_{i=r+1}^{2 r} X_{i}, \sum_{i=2 r+1}^{3 r} X_{i} \ldots \ldots$. represent the lapse of time consecutively between every $\mathrm{r}^{\text {th }}$ failure. A control chart for times between every $\mathrm{r}^{\text {th }}$ failure would throw more on the out of control signals than that of inter failure times. Xie et al. (2002) [1] named such a control chart as $t_{\mathrm{r}}$ control chart and developed control limits using the sampling distribution of $\sum_{i=1}^{r} X_{i}$. They have taken the example of exponential distribution and used the theory that the sum of exponential variates is a gamma variate to get the percentiles of $t_{r}$ control chart with the help of cumulative Poisson summations. If the inter failure times are not exponentially distributed, the control limits of $\mathrm{t}_{\mathrm{r}}$ chart of Xie et al. (2002) [1] cannot be used. To overcome this drawback Kantam and Ravi Kumar (2013) [7] suggested the following alternative approach to get control limits of $\mathrm{t}_{\mathrm{r}}$ chart for any distribution.

If $\left(X_{1}, X_{2}, \ldots X_{r}\right) ;\left(X_{r+1}, X_{r+2} \ldots X_{2 r}\right) ;\left(X_{2 r+1}, X_{2 r+2} \ldots X_{3 r}\right)$; etc are regarded as independent samples of size $r$ each, i.i.d random vari- 
ables having $\mathrm{F}(\mathrm{x})$ as their common model, then $Y_{1}=X_{1}, Y_{2}=\sum_{i=1}^{2} X_{i}, Y_{3}=\sum_{i=1}^{3} X_{i}, \ldots Y_{r}=\sum_{i=1}^{r} X_{i}$ becomes an or dered sample of size $r$ representing the time to first failure, time to second failure, $\ldots$, time to $\mathrm{r}^{\text {th }}$ failure respectively. $\mathrm{Y}_{\mathrm{r}}$ is the highest order statistic in an ordered sample $Y_{1}<Y_{2}<\ldots Y_{r}$. Thus, the $\mathrm{t}_{\mathrm{r}}$ chart is the control chart with $Y_{r}$ as the points on it representing the time to every $r^{\text {th }}$ failure. Therefore, when $r$ is fixed, the percentiles of highest order statistic in a sample of size $r$ would serve the purpose of control limits for the $t_{r}$ chart.

We know that $[F(x)]^{r}$ is the cumulative distribution function of $\mathrm{r}^{\text {th }}$ order statistic in a sample of size ' $r$ ' for the model $F(x)$. Hence the percentiles of $\mathrm{t}_{\mathrm{r}}$ chart with 0.9973 coverage probability would be the solutions of $[F(x)]^{r}=0.99865$ and $[F(x)]^{r}=0.00135$. The central line of the $\mathrm{t}_{\mathrm{r}}$-chart would be the solution of $[F(x)]^{r}=0.5$.

In the present paper, for the sake of convenience we develop the trcontrol limits for $r=2$. Adopting the concept of $t_{r}$ control chart for $\mathrm{r}=2, \mathrm{t}_{2}$ control chart we have grouped the 50 observations of Aarset (1987) [9] into 25 disjoint successive subgroups of size 2 each. Using the percentiles of highest order statistic in a sample of size 2 , the control limits of $t_{2}$ chart for the two probability models IRD and IHLD are given in Table 3.

Table $3: \mathrm{t}_{2}$ control Limits for IRD and IHLD

\begin{tabular}{|c|c|c|c|}
\hline Probability Model & LCL & CL & UCL \\
\hline IRD & 0.34061 & 1.05164 & 23.74143 \\
\hline IHLD & 0.99355 & 6.55046 & 2906.47794 \\
\hline
\end{tabular}

The sum of two observations in each successive subgroup of Table 2 shall become a point on $t_{2}$ chart with the above control limits. Table 4 gives the spread of the 25 points on $t_{2}$ chart.

Table 4: Subgroup observation for $\mathrm{t}_{\mathrm{r}}$ chart, $\mathrm{r}=2$

\begin{tabular}{|c|c|c|c|}
\hline S.No. & $\begin{array}{c}\text { Time to accumulate } \\
\text { of two failures }\end{array}$ & S.No. & $\begin{array}{c}\text { Time to accumulate } \\
\text { of two failures }\end{array}$ \\
\hline 1 & 0.3 & 14 & 115 \\
\hline 2 & 2 & 15 & 126 \\
\hline 3 & 2 & 16 & 134 \\
\hline 4 & 3 & 17 & 134 \\
\hline 5 & 9 & 18 & 147 \\
\hline 6 & 18 & 19 & 161 \\
\hline 7 & 30 & 20 & 165 \\
\hline 8 & 36 & 21 & 168 \\
\hline 9 & 36 & 22 & 169 \\
\hline 10 & 53 & 23 & 170 \\
\hline 11 & 76 & 24 & 170 \\
\hline 12 & 91 & 25 & 172 \\
\hline 13 & 97 & & \\
\hline
\end{tabular}

\section{Conclusion}

On critical comparison of the failure data with the control limits given in Table 2.2, it is observed that, with reference to IRD out of 50 observations the number of points falls below LCL is 2 and above UCL is 37 and between UCL and LCL is 11 where as in the case of IHLD the number of points falls below LCL is 2 and above UCL is 0 and between LCL and UCL is 48 .

The number of lifetimes below LCL is same for both the probability distributions Inverse Rayleigh and Inverse Half Logistic. Therefore, the early detection of wrong signal is equal in both the probability models. The number of lifetimes above UCL are more in IRD, which means that, with respect to IRD the lot is of better quality.

For $t_{r}$ control chart, the control limits given in Table 3.1, it is observed that, with respect to IRD, out of 25 observations the number of points falls below LCL is 1 and above UCL is 19 and be- tween UCL and LCL is 5 where as in the case of IHLD the number of points falls below LCL is 1 and above UCL is 0 and between UCL and LCL is 24.

The number of lifetimes below LCL for $t_{2}$ chart is same for both the probability models IRD and IHLD. Therefore, the early detection rate is equal in both the probability models. More number of points above UCL are observed in IRD hence IRD is preferable for $t_{2}$ chart when compared with IHLD.

To conclude the data for individual observations and for subgroup of size 2 IRD is noticed as a better model than IHLD.

\section{Acknowledgements:}

The authors are graceful to the referees for giving comments to improve our peace of research work. Also, we are very much thankful to the management of SRKR engineering college for their support and encouragement for publishing this research paper.

\section{References}

[1] Xie, M., Goh, T.N. and Ranjan, P. (2002). "Some Effective Control Chart Procedures for Reliability Monitoring”, Reliability Engineering and System Safety, 77, 143-150.

[2] Tadikamalla, P.R. and Popescu, D.G. (2007). "Kurtosis correction method for $\bar{X}$ and $\mathrm{R}$ control charts for long-tailed symmetrical distributions". Naval Research Logistic, 54, 371-383.

[3] Subba Rao, R. and Kantam, R.R.L. (2008). "Variable Control Charts for process mean with reference to double exponential distribution". Acta Cinica Indica, 34, 4, 1925-1930.

[4] Ravi Kumar, M.S. and Kantam, R.R.L. (2010) "Time Control Chart-Some IFR Models", Proceedings of the International Congress of Mathematicians Satellite International Conference on Probability and Statistics, Sambalpur University, India, September 1-3.

[5] Chaitanya Priya, M. (2011). "Kurtosis correction method for variable control charts-a comparison in Laplace distribution". Pakistan Journal of Statistics and Operations Research, VII, No. 1, 51-54.

[6] Srinivasa Rao, B, Pratapa Reddy, J., and Sarath Babu, G. (2012). "Extreme Value Charts and Analysis of Means (ANOM) based on the log-logistic distribution". Journal of Modern Applied Statistical Methods, 11, 2, 493-505.

[7] Kantam, R.R.L. and Ravi Kumar, M.S. (2013). "Time Control Chart-Burr Distribution". Collection of Recent Statistical Methods and Applications, A Publication of Department of Statistics, University of Kerala, 307-314.

[8] Subba Rao, R, Pushpa Latha Mamidi, M.S. Ravi Kumar. (2016). "Skewness Corrected Control Charts for two Inverted Models". Journal of Chemical and Pharmaceutical Sciences. 10, 51-55.

[9] Aarset, M.V. (1987). "How to identify bathtub hazard rate". IEEE Transactions on Reliability, R-36, 106-108. 